\title{
JARMUSCH VS REAGAN. GOLPE AL SUEÑO AMERICANO ${ }^{1}$
}

\author{
Gabri Ródenas ${ }^{2}$
}

Resumen: El presente artículo trata de analizar la relación tensa entre el cine realizado por Jim Jarmusch en la década de los 80 y el cine hegemónico durante lo que podríamos denominar el "Periodo Reagan", caracterizado por un cierto infantilismo, un intento de recuperar el espíritu triunfalista estadounidense, ahuyentando el "Síndrome de Vietnam", y salvar los restos del sueño americano. Frente a este tipo de cine, Jarmusch aboga por un cine del insomnio. Propuesta que comenzaría a verse truncada a partir de 1991 con el estreno de Night on Earth.

Palabras clave: Jarmusch, Reagan, Vietnam, cne independiente, statu quo, Hollywood, seño americano.

\section{Jarmusch vs Reagan. A Blow to the American Dream}

Abstract: The present paper analyzes the strained connection between Jim Jarmusch's Cinema during the 80 's and the hegemonic cinema in what we could call "The Reagan in Office", characterized by a certain infantilism, a recovery of the triumphalist North American Spirit attempt, frightening the "Vietnam Syndrome" away, and saving the rests of the American Dream. Opposite this kind of cinema, Jarmusch advocates a Cinema of Insomnia. Proposal that would start to be truncated by 1991, with the release of Night on Earth.

Key words: Jarmusch, Reagan, Vietnam, independent cinema, status quo, Hollywood, American dream.

\section{INTRODUCCIÓN}

El germen de lo que podríamos denominar "primera etapa" del cine de Jim Jarmusch, y que recorrería el periodo comprendido entre Permanent Vacation (1980) y Night on Earth (Noche en la Tierra, 1991), coincide con un margen de tres años, con el mandato de Ronald Reagan, de 1981 a 1989. La Guerra Fría llegaba a su fin, pero no por ello podemos decir que la situación internacional fuera estable. La naturaleza del armamento tanto estadounidense como soviético hizo que se acuñase el término MAD (Mutual Assured Destruction) ${ }^{3}$. El equilibrio, por tanto, era irreal. Reagan impulsó el desarrollo de escudos contra armas nucleares enemigas - que serían retomados por Bush Jr. posteriormente- de tecnología láser y misiles interceptores. Todo eso sería denominado "Guerra de las galaxias", sin duda mo-

1 Date of reception: April 2009.

Date of acceptance and final version: July 2009.

2 Director de la sección de Filosofía de la Revista Oficial de la Universidad de Murcia, Universidad de Murcia; $\triangle$ gabrirodenas@gmail.com.

3 No incidiremos en el posible juego de palabras al que da pie el término MAD (loco). 
tivado por la película homónima. Reagan, a pesar de ello, pareció devolver el ánimo a una América todavía sujeta al fantasma de Vietnam, constituyendo de nuevo una nación fuerte y anunciando justamente el fin de ese "Síndrome de Vietnam". Esto puede verse en ejemplos tardíos, muy próximos a la era Reagan, en obras como The Deer Hunter (El cazador), de Michael Cimino (1978), Apocalypse Now, de Coppola (1978) y en otros trabajos ya realizados durante su mandato: First Blood (Acorralado, Ted Kotcheff, 1982) y Rambo: First Blood II (Rambo, George P. Cosmatos, 1985), Missing in Action (Desaparecido en combate, Joseph Zito, 1984) y su secuela de 1985 (dirigida por Lance Hool), Platoon (Oliver Stone, 1986), entre otros títulos. The Deer Hunter, no obstante, es la primera película con relativo presupuesto que aborda la guerra de Vietnam. Con anterioridad se habían realizado otros films, pero sin tanta difusión ni presupuesto ${ }^{4}$. Esto tendría, como se verá más adelante, su clara proyección cinematográfica en obras como la serie First Blood. Otros autores, como Robin Wood y Breixo Viejo, por el contrario, hacen un balance más desfavorable 5 . Ambos coinciden en la decadencia y mayor índice de estupidez que caracterizó su mandato. Sirva la siguiente cita como indicador de esta posición: "Al reflexionar sobre los años ochenta, el crítico de arte Robert Hughes aseguraba que, para definir la situación en la que el reaganismo había sumido a los Estados Unidos, sólo había un sustantivo: "decadencia".

Con la eficacia de un sonámbulo -señaló Hughes- [Reagan] educó a América hasta rebajarla a su nivel. Dejó a su país en 1988 un poco más estúpido que en 1980 y mucho más tolerante con las mentiras, porque su estilo de presentar las imágenes cortaba el vínculo entre las ideas y estimulaba la derrota del pensamiento. (Viejo 2001: 18-19) ${ }^{6}$

La argumentación de Wood tiene más que ver con la producción de películas orientadas al entretenimiento, lo que, a su juicio, contribuyó a eludir cuestiones más fundamentales y tuvo el efecto de un narcótico. Asimismo, su análisis se centra en la crítica de los aspectos orientados a mantener el statu quo (patriarcado, primacía del hombre blanco, heterosexual, la familia nuclear, etc. $)^{7}$.

Nuestro interés no es, obviamente, ni posicionarnos con respecto al gobierno de Reagan ni recrear ese momento histórico. Lo que pretendemos, repetimos, es analizar el tipo de cine que por aquel entonces comenzó a hacerse y ver hasta qué punto el cine de Jarmusch surge como una suerte de reacción.

\footnotetext{
4 Agradecemos a Amaya Muruzábal permitirnos reproducir fragmentos de su tesis doctoral, aún inédita, sobre La representación cinematográfica del regreso. El primer capítulo es un resumen magnífico de los antecedentes del conflicto en Vietnam y aporta numerosas claves para entender el tratamiento fílmico del mismo. Nos sentimos también muy agradecidos hacia Susana Torrado, que hizo las veces de amable "intermediaria".

5 Véase Wood 2003 y Viejo 2001.

6 El texto de Hughes al que hace referencia es La cultura de la queja. Trifulcas norteamericanas. Barcelona: Anagrama, 1994. Jameson, refiriéndose al asentamiento de la cultura posmoderna estadounidense, afirma: "Pero éste es el momento de llamar la atención del lector sobre algo obvio: a saber, que toda esta cultura posmoderna que podríamos llamar estadounidense, es la expresión interna y superestructural de toda una nueva ola de dominación militar y económica norteamericana de dimensiones mundiales: en este sentido, el trasfondo de la cultura lo constituyen la sangre, la tortura, la muerte y el horror" (1984: 19). Las cursivas son nuestras. Teniendo en cuenta que nos referimos a un texto de 1984, es fácil ver a qué nueva ola de dominación se está refiriendo el autor.

$7 \quad$ A este respecto véase también Pfeil 1998.
} 
Juan Antonio Suárez afirma que, tal y como Robert Frank retrata la otra América de Eisenhower, Jarmusch hace lo mismo con la de Reagan ${ }^{8}$. Es importante hacer aquí un matiz. Cierto que Jarmusch retrata el otro lado de la sociedad norteamericana de los 80, por así decirlo, el insomnio americano en lugar del sueño americano -en este sentido podemos afirmar que no es el Rockwell del cine, sino el Edward Hopper (o el Eric Fischl, aunque en menor grado)- pero no hay carga política explícita. O, citando a Breixo Viejo:

La América que presenta Jarmusch no es el país de las oportunidades ni de las grandes promesas de reaganismo; es la América del Insomnio Americano, una tierra helada y fantasmal, una playa baldía, un paisaje arruinado por las fábricas, un territorio por el que los personajes vagan sin rumbo en busca de un destino prometido e inexistente, más extraño que el paraíso: la América del Sueño Americano. (Viejo 2001: 78) ${ }^{9}$

A diferencia de otros directores del momento como Spike Lee o los integrantes del Cinema of Transgression, Jarmusch no desarrolla un discurso de orden ideológico explícito. Su cine es esencialmente apolítico (al menos hasta Dead Man, que se sitúa fuera de los límites nuestro estudio). Por apolítico queremos decir no adscrito a ninguna ideología política vigente: ni la de Reagan ni la de sus detractores más enérgicos. Obviamente, todo cine ( $u$ obra de arte en general) presenta una serie de elementos ideológicos, aunque sea de modo implícito. En el caso de nuestro director, él mismo nos da información sobre su postura: "Tengo fuertes convicciones políticas, pero no siento que mi trabajo sea al lugar donde manifestarlas"10 (en Hertzberg 2001: 97.). Con el fin de aclarar bien este extremo, repetimos que nuestro objetivo aquí es desvincular la obra de Jarmusch del panfleto. En este sentido, podemos afirmar que la conexión tiene un carácter básicamente cronológico: se dieron en la misma franja temporal, pero, si bien sí entraña -y de un modo determinante- una reacción contra el cine de la época (contra lo que representaba), no supone un ataque directo contra la política de Reagan. De hecho, su obra tiene un cariz más humano y poético que político en general.

\section{LA DOBLE CARA DEL CINE NORTEAMERICANO DURANTE EL PERIODO REA- GAN}

Los años 80 en el terreno cinematográfico se caracterizaron por una cierta polaridad: por un lado, se produjo un número ingente de películas orientadas al entretenimiento en sentido más estricto y, por otro, el auge de géneros extremadamente violentos y con alto contenido sexual, a modo de contrapunto meramente estilístico, dado que, como veremos, en ambos casos hay un deseo de preservar el statu quo.

Por lo que respecta a las primeras, no obstante, podríamos llegar a decir que se pidió al público adulto que mirase a la pantalla con ojos de niño ${ }^{11}$, algo que ya se había solicitado

\footnotetext{
Véase Suárez Sánchez 2007: 36.

Véase también Hernández Ruiz 2003.

10 Procede de la entrevista concedida a Luc Sante.

11 Sobre esta cuestión, véase Wood 1986: 145-147; Muruzábal op.cit.: 271 y Cook 1998. Lewis. op.cit .II-37.
} 23. 
con Jaws (Tiburón, Steven Spielberg, 1975) o la Star Wars (Guerra de las galaxias, George Lucas, 1977). Alcanzó el paroxismo en la década de los 80 con trabajos como Porky's (Bob Clark, 1982) -que contaba con antecedentes como Animal house (Desmadre a la americana, John Landis, 1978)-, sus dos secuelas y sus numerosos imitadores -Up the Creek (Los albóndigas en remojo, Robert Butler, 1984)-, así como otras películas de temática nerd y universitaria en general. Ejemplos del infantilismo made in USA también son la serie de Revenge of the nerds (La revancha de los novatos, Jeff Kanew, 1984) o films más (aparentemente) inocentes del estilo The Goonies (Los Goonies, Richard Donner, 1985), E.T. (Steven Spielberg, 1982), Raiders of the Lost Ark (Indiana Jones: En busca del arca perdida, Steven Spielberg, 1981. La secuela de 1984 dirigida igualmente por Spielberg) ${ }^{12}$, Back to the Future (Regreso al futuro, Robert Zemeckis, 1985) y sus secuelas, Ghostbusters (Los cazafantasmas, Ivan Reitman, 1984) -que también contó con entregas posteriores-. El género de ciencia ficción -Short Circuit (Cortocircuito, John Badham 1986)- o de pseudo-terror para niños y adolescentes como Gremlins (Joe Dante, 1984) -y sus múltiples emuladores, como Critters (Stephen Herek, 1986)- también tuvo su importancia a la hora de difundir el estilo de vida americano.

La lista de trabajos de este tipo es verdaderamente extensa, si bien todos ellos presentan elementos en común, tales como dinamismo, modo de vida urbano, hedonismo, individualismo, despreocupación, búsqueda de un estilo cool (traducible por los modismos "guay", "molón"), desenfado... "Si el cine de los 80 destacó por algo fue por su colorido y sus aires casi festivos" (Guillén y Puente 2008: 153). Los personajes de todas estas películas comparten y defienden el estilo de vida americano y exhiben un cierto infantilismo. Wood sostiene que ese infantilismo se encuentra ligado al deseo de no asumir responsabilidades (1986: 147). El imperio de lo novedoso también es señalado por Wood, "New is Good" (1986: XXIII-XXV).

En muchas de las producciones de los 80 el componente sexual, como se ha comentado, era verdaderamente elevado, rozando lo que suele denominarse softcore. No eran escasos los trabajos que mezclaban sexo, lenguaje malsonante con extrema violencia, tal y como sucedía en la mayor parte de los trabajos protagonizados por Charles Bronson en aquella época, añadiendo un grado de violencia a los trabajos anteriores de Clint Eastwood, tales como las entregas de Harry sobre todo e incluso a las protagonizadas por el propio Bronson una década antes como, por ejemplo, Death Wish (El justiciero de la ciudad, Michael Winner, 1974). Éstas seguían la línea argumental del héroe dudoso que limpia las calles de basura humana, tal y como llevase a cabo Travis Bickle (Taxi Driver, Martin Scorsese, 1976) ${ }^{13}$. Con características similares encontramos películas como The Exterminator (El exterminador, James Glickenhaus, 1980), que además cuenta con el aliciente de tener como protagonista a un veterano de Vietnam (al igual que Travis).

Tanto los films bélicos como la serie First Blood o Missing in Action, las películas orientales y occidentales de artes marciales y ninjas, o los trabajos más urbanos, como los últimos citados, se comparte el mismo esquema: héroe solitario que ha sido ultrajado y

12 Como quedará patente sobre todo en las obras de terror, los 80 consolidaron la era de las secuelas o, como Wood afirma, la era de "secuelas y repetición" (1986: 144). El propio Jarmusch afirmó que "era una plaga en Hollywood" (en Carr 1987).

13 La "Excremental City", como es denominada por Wood 1986: 46 y ss. 
decide tomarse la justicia por su mano. Dependiendo del género, el enemigo también presenta unas características concretas: el enemigo bélico de turno; los proxenetas, drogadictos, pederastas, maleantes y gentes de mal vivir; el sempiterno enemigo oriental, simbolizado por la mafia china o la yakuza japonesa, etc.

En última instancia observamos que todos estos trabajos buscan mantener el statu quo. Las películas mencionadas son trabajos tan conservadores como la política de Reagan y buscan claramente volver a construir un espíritu americano libre/liberador, justo y triunfador -figura, por cierto, clave en el cine de la época-. En esta línea, surge un nuevo grupo de superhéroes, contrastando con los personajes de los films de los 70, cuya función queda perfectamente reflejada en esta observación de Robert Towne:

Durante gran parte de los años setenta, el objetivo era revelar la disparidad entre lo que el país afirmaba ser y lo que los directores percibían. Y había un público interesado en esa cuestión. Cuando llegaron los ochenta, entramos en un mundo de súper-héroes de esteroides. Empezando por Superman, Sly, Arnold, e incluso Bruce Willis volvieron a hacer la guerra de Vietnam, y la ganaron. (Muruzábal 274)

O en la siguiente de Fred Pfeil "[Rambo] quien lucha y gana una segunda Guerra de Vietnam por sí mismo en el año espantoso de la reelección de Reagan" (Pfeil 1998: 148).

Como puede verse, se trata de otro de los mecanismos para disipar ese fantasma de Vietnam e invertir la situación. A la luz del revisionismo reaganiano, realmente el nuevo esquema sería algo similar a: la Guerra de Vietnam fue una guerra justificada y, retrospectivamente, apelando al nuevo orden moral, fue ganada por los Estados Unidos. Esos nuevos héroes, por tanto, restaurarían y encarnarían la validez de los "Good Old Values". Wood por su parte arremete contra esos "valores", identificándolos con el fascismo, el racismo, el sexismo y el capitalismo "democrático" (1986: 147).

Con menos esteroides, con las mismas características y con un mayor sex appeal de cara al público femenino, surgen los héroes de las sagas Lethal Weapon (Arma letal, la primera dirigida por Richard Donner en 1987) y Die Hard (La jungla de cristal, la primera dirigida por John McTiernan en 1988) ${ }^{14}$. Los personajes encarnados por Mel Gibson y Bruce Willis respectivamente tratarán de defender los viejos valores con la dificultad añadida de la nueva situación de la mujer, ocupando prácticamente los mismos altos cargos y puestos de responsabilidad que el hombre y compartiendo, o incluso superando, su estatus social y económico. Gibson y Willis asumen esa nueva masculinidad: los músculos siguen estando presentes, pero una nueva sensibilidad debe aflorar.

Como es fácil de imaginar, dicho cambio es meramente estratégico. En última instancia, se insinúa una suerte de reproche: "Mira lo que pasa cuando [tú, mujer] dejas el hogar y abandonas los viejos valores tradicionales". El final feliz está asegurado y dicha felicidad es generada por la vuelta al statu quo ante.

Durante esos años siguió explotándose el sistema Blockbuster (éxito de taquilla), ya instalado en los 70 a raíz de éxitos como The Godfather (El Padrino, Francis Ford Coppola,

14 Sobre estas dos sagas, véase el magnífico artículo ya mencionado de Fred Pfeil. 
1972) o Jaws ${ }^{15}$. Aunque compartiendo escena con las mencionadas producciones orientadas directamente al vídeo o, sencillamente, con menos pretensiones.

\section{UN CINE MUY RENTABLE: EL NUEVO CINE INDEPENDIENTE}

También a principios de los 80 comenzaron a surgir festivales dedicados al cine independiente (Sundance, impulsado por Robert Redford en 1983 o los Independent Spirit Awards, fundado en 1984 con el nombre de FINDIE ("Friends of Independents") ${ }^{16}$. Estos festivales se posicionaban como alternativas a la mentalidad blockbuster ${ }^{17}$. Es importante señalar un aspecto: "Mientras que esas películas variaban con respecto al producto dominante de Hollywood, [los festivales independientes] seguían trabajando con la lógica del sistema dominante" (Kleinhans 1998: 313), limitándose a ocupar los márgenes temáticos, por así decirlo, que la gran industria descuidaba o desatendía. No hay que olvidar que, proporcionalmente, trabajos como Sex, Lies and Videotape (Sexo, mentiras y cintas de vídeo, Steven Soderbergh, 1989) o la propia Stranger than Paradise (Extraños en el paraíso, Jim Jarmusch, 1984), cuyo beneficio multiplicó por veinte su coste, fueron muy rentables. Esto abriría el camino a muchos otros independientes, hasta su colapso en torno a mediados de los 90, cuando las pequeñas productoras fueron absorbidas por las grandes, convirtiéndose en minimajors, y grandes majors (salvo Disney), a su vez, fueron absorbidas por multinacionales ${ }^{18}$.

En este caldo de cultivo o nicho ecológico -tomando prestada la expresión Ian Hacking-surge la figura de Jim Jarmusch. Frente a las estructuras lineales y de trama fuerte comunes a todos los films del momento (los de difusión masiva, por supuesto), Jarmusch se lanza a explorar el terreno de la fragmentación, la elipsis, la trama débil ("narración débil") o relato de "en medio" al que se refiere Breixo Viejo. El cambio estilístico, según Viejo, entraña un cambio temático y, por extensión, de orden moral: "Su discurso, construido en sentido inverso (por medio y no por los extremos), da la vuelta a la ideología adscrita a la convencionalidad de la narración e intercambia al sueño por el insomnio y al héroe por el perdedor. Se trata, en definitiva, de un contradiscurso (sic.)" (Viejo 2001: 63). Todo este procedimiento tiene por finalidad la "desdramatización" del relato. Las comillas son acertadas, dado que no podemos hablar de desdramatización total, sino de substitución de un tipo de dramatización por otra, lo que refuerza nuestra tesis de la renovación partiendo de lo dado. Por estos motivos, en ocasiones emplearemos el término "redramatizar". Al igual que sucede, por ejemplo, en el cine de Ozu, los "tiempos muertos", el gesto nimio, los pequeños detalles, los actos "triviales" y situaciones cotidianos encierran una tensión. Desdramatizando el relato de ese modo, Jarmusch da prioridad a otros elementos más marginales en el cine tales como la magia de lo cotidiano y su importancia como nexo

\footnotetext{
15 El primer capítulo del texto de Muruzábal es un buen documento para aquel que desee profundizar en la génesis del sistema blockbuster. Véase también, sobre el origen de los blockbusters y los "Hollywood Brats" (Francis Ford Coppola, George Lucas, Steven Spielberg, Martin Scorsese, Brian de Palma) Cook 1998: 12-35 y Corrigan 1998 sobre la nueva política de autor en Hollywood y el sistema blockbuster/high concept, así como el artículo de Wyatt 1998.

16 En 1986 cambió de nombre por el de Independent Spirits Awards.

17 Véase Corrigan 1998 y Kleinhans 1998.

18 Véase Augros 2000 y Weinrichter 2003.
} 
humano o el retrato de los marginados, concretamente de los marginados existenciales. Por estos motivos afirmamos que su discurso no es político - politizado-sino humano. En este sentido, concedemos que el cine de Jarmusch presenta una fuerte impronta moral, pero, y esto es fundamental, no moralizante.

\section{COOL INDIE}

Podemos ahora tratar de razonar hasta qué punto el cine de Jim Jarmusch supone una reacción con respecto al cine hegemónico o típico de los 80 , teniendo en cuenta esa duplicidad o doble cara. En primer lugar, y como ya se ha señalado, Jarmusch se desmarca del contexto estrictamente político e ideológico de Reagan. Su mirada tiene otros objetivos. Cuestiones como la Guerra de Vietnam no tienen cabida en su filmografía, salvo por la aparición de un personaje muy secundario en la malograda Permanent Vacation, el típico sin techo que afirma ser un veterano de Vietnam, cuya función en el film es más de desahuciado que de ex-combatiente. Más presencia tienen cuestiones como el desempleo. Podemos verlo en los personajes de Down by Law (Bajo el peso de la ley, Jarmusch, 1986), los cuales no desarrollan trabajos regulares y, mucho menos, lícitos. Y, de manera evidente, en Stranger than Paradise. Pensemos en la escena en la que los dos protagonistas masculinos deciden viajar a Cleveland. Preguntan a un tipo que espera un autobús que lo lleve al trabajo y le preguntan qué camino deben tomar para llegar hasta allí. Teniendo en cuenta que se hallan en Nueva York, el señor lo toma como una burla y su respuesta no puede ser más contundente: "No me vaciles, tío, que voy a trabajar". Permítasenos transcribir el resto de la conversación:

WILLY: ¿Dónde trabajas?

TIPO: En una fábrica

WILLY: [A Eddie] Vámonos

EDDIE: Joder Willy, no debiste vacilarle de ese modo. ¿Te imaginas trabajar en una fábrica?

WILLY: No soy capaz. Ahora me siento mal.

EDDIE: No te sientas mal.

Nuestros personajes rechazan así el sueño americano y también la estructura convencional señalada por Wood o Pfeil. Cierto que Willy es blanco y heterosexual, pero también es un inmigrante húngaro, no tiene familia ni trabajo, ni la menor expectativa. Tampoco aquí encontramos un debate político respecto a la cuestión de la situación laboral de los norteamericanos en 1980 (1984 concretamente). Ambos personajes asumen su posición y no desean en absoluto modificarla. No hay discurso político. Sólo una crónica distanciada. No se pretende encubrir una defensa de los valores tradicionales y la apuesta clara es por el perdedor. En este fragmento se aprecia en dos niveles: lástima por el perdedor funcional reflejado en el trabajador de fábrica y defensa del propio estatus de perdedor de los dos deadbeats que protagonizan la historia.

El cine de Jarmusch no se orienta en ningún caso al divertimento banal. En esta dirección podemos recordar las palabras de Cassavetes "No hay nada que deteste más que la 
idea de que me entretengan" o "Nunca diré que lo que hago es entretenimiento" (Carney 2001: 471). No recurre al efectismo visual gracias a los efectos especiales ${ }^{19}$; no hay sexo ni sangre; no nos invita a mirar el mundo con ojos de niño (en el sentido de Spielberg, Donner o Lucas).

Obviamente sí apreciamos una construcción de los personajes totalmente diferente de la estándar. Frente al bueno-bueno o malo-malo del cine clásico ${ }^{20}$ (con notables excepciones) o de los años 80, Jarmusch elabora una tipología híbrida, ambigua, muy en la línea de Godard o Bresson. "Y en su extremo jansenismo, Bresson muestra la misma infamia del lado de las obras, es decir, del lado del mal y del bien" (Deleuze 1984: 167). Esta afirmación, referida a los hechos, puede extenderse, como es fácil de suponer, a los personajes. No nos extenderemos demasiado ni aquí ni más adelante sobre esta cuestión, pero no queremos dejar de señalar la enorme relación que hay entre jansenismo y taoísmo, algo que, en el caso de Bresson -en un cierto sentido tan oriental- es notable y pertinente por nuestra parte el apuntarlo. Sí es importante matizar que Bresson, sujeto a una tradición cristiana, carga con el lastre trágico de la predeterminación como algo doloroso, algo extraño al pensamiento taoísta. En oposición a la figura del triunfador, sexy, dinámico, Jarmusch dibuja una serie de individuos perdedores, poco atractivos y decididamente apáticos, aunque sin caer en una "mitificación del marginal".

Es en plano técnico y lingüístico donde la diferencia se hace más notable. Ya hemos hablado del uso de largos planos, o planos-secuencia, y del estatismo de la cámara de Jarmusch, en abierto contraste con los planos de brevísima duración e intensidad en la mayor parte del cine de los 80. En lugar del dinamismo, él se decanta por la lentitud y la quietud.

Al mismo nivel que los personajes o la propia trama están los ambientes, escenarios y decorados, siempre naturales (esto es, no expresamente realizados para el film en cuestión). El peso de la historia queda disminuido y sustituido en parte por los tiempos muertos, los silencios. Frente al montaje lineal, un montaje elíptico, paralelo o asincrónico. Igualmente las transiciones no se ciñen al esquema tradicional: fundidos en negro para sugerir elipsis más largas o cambio temático; encadenados que nos informan de un intervalo más breve, simultaneidad o similitud temática, así como insinúan una visión diferente sobre el mismo asunto por parte de los distintos implicados. Las transiciones de Jarmusch muestran el vacío del tiempo, la banalidad, el vagabundeo interior del personaje y una exhibición de esos otros elementos no adscritos estrictamente a la diégesis (como el entorno). Resulta innecesario dejar claro que también Jarmusch usa convencionalmente las transiciones en múltiples ocasiones. Lo que queremos aquí es mostrar las diferencias y las nuevas aportaciones y no lo común.

En otro orden de cosas, Jarmusch se desmarca de la búsqueda del blockbuster o de la estrategia del high concept film, aliándose con el bando del indie de los 80 (y sólo de los 80 , matizamos). No es una cuestión de necesidad. La elección de temas, su particular uso del lenguaje fílmico, su negativa a dirigir guiones escritos por otros, su deseo de trabajar con el mismo equipo técnico y artístico nos da claras muestras de su elección personal por ese tipo de cine. A este respecto, Jarmusch ha hecho algunas declaraciones que no dejan

19 Sobre lo "escópico", véase Company, J.M. y J. M. Marzal 1999.

20 Sobre los problemas del cine clásico (como concepto) y del M.R.I, véase Gómez Tarín (2006: 20). 
de ser un ejercicio de ironía. En una conferencia a propósito de Dead Man, se le preguntó por qué no había hecho películas en Hollywood, a lo que él respondió: "porque nunca me han llamado" (en Viejo 2001:52). Pero no debemos tomar esta afirmación muy en serio. En esa misma rueda de prensa Jarmusch había declarado:

Toda esa idea del cine independiente me deja perplejo. Ya no sé qué significa. Solía tratarse de películas modestas realizadas sin mucho dinero y sin la interferencia de aquéllos a los que sólo interesaba obtener beneficios a partir del film.

Si tenemos en cuenta que Dead Man es de 1995, queda claro que hay un antes y un después en el cine independiente y dónde reside la diferencia. La situación de dicho cine en 1980 poco o nada tiene que ver con la de 1990, por ejemplo. Esto es algo que ya hemos apuntado repetidamente.

¿Qué es, pues, “Cine Independiente” para Jarmusch? Él nos da la respuesta:

Para mí "independiente" significa no dejar que tu trabajo sea dictado o configurado por las normas del mercado, lo cual no quiere decir que no dejes que tu película entre a formar parte de él. Cada vez que hago un largometraje, el dinero que empleo no es mío, así que, evidentemente, tengo que hacer consideraciones financieras, no soy ingenuo. Pero para mí el dinero sirve a la película y no la película al dinero tan pronto como el trabajo está a disposición del presupuesto y no el presupuesto a disposición del trabajo, se acaba la "independencia" 21 .

El dictamen no puede ser más contundente ${ }^{22}$. Por otra parte, sabemos que Jarmusch mantiene una serie de exigencias ante las productoras, tales como la posesión del negativo, supervisión directa de la distribución, revisión de los derechos cada siete años, etc. ${ }^{23}$. De todo esto se sigue que la decisión jarmuschiana es de orden moral.

Lo que nosotros encontramos en Jarmusch no es una oposición directa al establishment, sino mediante el rodeo. Casi podríamos decir que Jarmusch se sitúa al margen, en otro plano. No encontramos en él una crítica explícita tal y como lleva a cabo, por ejemplo, Tom Dicillo en muchos de sus films -Living in Oblivion (Vivir rodando, 1995), The Real Blonde (Una rubia auténtica, 1997) o Delirious (2006)- o Robert Altman en The Player (El juego de Hollywood, 1992). Para ser exactos, e independientemente de haber usado el término "margen" un poco más arriba, Jarmusch adopta la actitud de Cassavetes y al igual que éste, "en realidad es menos un marginal que un minoritario, es decir, alguien que desea sobre

${ }^{21}$ Citado en "Filmmaker Focus: Jim Jarmusch" (www.sundancechannel.com/focus/jarmusch/html, 1996). Un guiño en este sentido lo encontramos en Night on Earth cuando el taxista Helmut asegura que el dinero no es importante, que lo necesita, evidentemente, pero que no es lo principal para él que es un "payaso".

22 En diversas entrevistas Jarmusch rechaza la etiqueta de "Padrino de los independientes". Véase, por ejemplo, Rothenberg 2005: 223. De hecho, Jarmusch rechaza cualquier tipo de categorización. "Tratar de categorizar los movimientos artísticos o cualquier cosa es ridículo" (de la entrevista concedida a Tod Lippy y reproducida en Suárez Sánchez 2007: 158).

23 Sobre estas cuestiones y otras relativas a las cifras de producción y recaudación, véase el capítulo "Modes of Production" en David Lakly "A Sad and beautiful World: the Films of Jim Jarmusch, an American Independent Auteur" (Tesis no publicada). No hemos tenido acceso a ella, de modo que la información la hemos tomado de Viejo (2001: 56). 
todo, incluso antes de oponerse, construir su propia empresa al lado, y no a la sombra del gigante hollywoodiense, tratando por todos los medios de preservar su autonomía" (Jousse 1992: 161). Realmente su oposición a Hollywood, más que deberse a motivos políticos, tiene su raíz en las exigencias (y limitaciones) de orden estético que la gran industria impone $^{24}$. Actitud que, como puede verse a partir de 1991 con el estreno de Night on Earth, se ha visto suavizada hasta el extremo, hasta el punto de que podemos afirmar que, si bien de reojo, desde entonces no ha dejado de mirar la golosina hollywoodiense.

\section{REFERENCIAS BIBLIOGRÁFICAS}

Augros, J. 2000. El dinero de Hollywood. Barcelona: Paidós.

CASAS, Q., ed. 2003. Itinerarios al vacío. Madrid: T\&B Editores.

CARney, R., ed. 2001. Cassavetes por Cassavetes. Barcelona: Anagrama.

CARr, J. 1986. "Jim Jarmusch: A Little Nervous". The Boston Globe . 17 de septiembre.

Company, J.M, y J.M. Marzal. 1999. La mirada cautiva. Formas de ver en el cine contemporáneo. Valencia: Generalitat Valenciana.

Cook, D. 1998. "Auteur Cinema and the 'Film Generation' in 1970s Hollywood”. New American Cinema. Ed. J. Lewis. Durham and London: Duke University Press. 11-37.

Corrigan, T. 1998. "Auteurs and the New Hollywood". New American Cinema. Ed. J. LEwIS. Durham and London: Duke University Press. 37-63.

Deleuze, G. 1984. La imagen-movimiento. Barcelona: Paidós.

Gómez Tarín, F. J. 2006. Al final de la escapada. Barcelona-Valencia: Nau Llibres/ Octaedro.

Guillén, S. y A. Puente. 2008. 80 películas de los 80. Una lectura ácida. Madrid: T\&B Editores.

Hernández Ruiz, J. 2003. "Todo está en tránsito". Itinerarios al vacio. Ed. Q. CASAS. Madrid: T\&B. 33-43.

HertzBerg, L., ed. 2001. Jim Jarmusch. Interviews. Mississippi: University Press of Mississippi.

JAMESON, F. 1984. El posmodernismo o la lógica cultural del capitalismo avanzado. Barcelona: Paidós.

Jousse, T. 1992. John Cassavetes. Madrid: Cátedra.

\footnotetext{
24 Véase Rothenberg (2005: 223). En la entrevista concedida a Rothenberg afirma que sí hay algunos factores "políticos" (política de "empresa") que le mueven a rechazar los dictámenes de Hollywood. Pero, repetimos, sus motivaciones son de orden estético. Dicha entrevista es un excelente documento a la hora de estudiar el punto de vista, menos radical del que pueda suponerse, de Jarmusch sobre Hollywood y que reafirma la vinculación con los planteamientos de Cassavetes.
} 
Kleinhans, C. 1998. "Independent Features: Hopes and Dreams". New American Cinema. Ed. J. Lewis. Durham and London: Duke University Press. 307-327.

Lewis, J. 1998. "Money Matters: Hollywood in the Corporate Era". New American Cinema. Ed. J. Lewis. Durham and London: Duke University Press. 87-121.

Press.

. ed. 1998. The New American Cinema. Durham y London: Duke University

Patterson, C., ed. 2005. Captured: A film/Video history of the lower east side. New York: Seven Stories Press.

Pfeil, F. 1998. "From Pillar to Postmodern: Race, Class, and Gender in the Male Rampage Film". New American Cinema. Ed. J. Lewis. Durham and London: Duke University Press. 146-186.

Rotenbergh, J. 2005. "(Dead) panning Through the Lower East Side with Jim Jarmusch". Captured. Ed. C. Patterson. New York: Seven Stories Press. 221-232.

SuÁrez SÁnchez, J. A. 2007. Jim Jarmusch. Urbana and Chicago: University of Illinois Press.

VIEJo, B. 2001. Jim Jarmusch y el sueño de los justos. Madrid: Ediciones JC.

Weinrichter, A. 2003. "Lejos de Hollywood”. Itinerarios al vacio. Ed. Q. Casas. Madrid: T\&B. 13-19.

Wood, R. 1986. Hollywood from Vietnam to Reagan... And beyond. New York: Columbia University Press.

Wyatt, J. 1998. "Marketing and Distribution”. New American Cinema. Ed. J. Lewis. Durham and London: Duke University Press. 64-86. 